\title{
PROPUESTA METODOLÓGICA PARA LA GESTIÓN DE INVENTARIOS EN UNA EMPRESA DE BEBIDAS POR EL MÉTODO JUSTO A TIEMPO CASO DE ESTUDIO: ABASTECIMIENTO DE AZÚCAR
}

\author{
Richard Javier Vélez Rojas ${ }^{1}$ \\ Giovanni Pérez Ortega ${ }^{2}$
}

\begin{abstract}
RESUMEN
El artículo plantea una propuesta metodológica que tiene como fin mejorar el manejo de inventarios en una compañía de bebidas, utilizando en este caso una de las materias primas más importantes como lo es el azúcar. A partir de problemas que se encontraron con el abastecimiento de dicha materia prima, se desarrolló en cinco fases, que se deben tener en cuenta para un óptimo manejo de inventarios, estas fases son un paso a paso que explica y muestra las condiciones necesarias para desarrollar el método, qué partes son las involucradas y cuál es su función en cada uno de los literales del proyecto; adicional a esto se describen fórmulas que ayudan a la planeación, el control y ejecución apropiada para el proyecto.
\end{abstract}

\section{PALABRAS CLAVES}

Justo a Tiempo, Cadena de Abastecimiento, Inventarios, ERP, Stock de seguridad.

\begin{abstract}
This article presents a methodological proposal, which aims to improve inventory management in a beverages company, using sugar as the most important raw material in this case. Because of some problems found with the supply of the aforementioned raw material, 5 phases were developed in order to guarantee an optimum inventory management, the phases are stages that explain and show the necessary conditions to develop the method, they also explain which parts are involved and what is their function in each of the project headings, plus they describe formulas that help with planning, control and appropriate execution for the project.
\end{abstract}

\section{KEYWORDS}

Just in Time, Chain of Supply, Inventory, ERP, Security Stock 


\section{INTRODUCCIÓN}

En mercados altamente competitivos, es importante garantizar la satisfacción del cliente, con el fin de lograr aumentar la participación de la compañía en el mercado o tratar de sobrevivir en éste. Por esta razón, para poder cumplir con la demanda que exige el mercado, la administración adecuada de la cadena de abastecimiento es primordial para las empresas, ya que el propósito es lograr una relación integrada entre clientes y proveedores. Esta adecuada gestión nos puede llevar por el camino de la competitividad, lo cual podría abrir el horizonte del negocio hacia compradores potenciales que pueden estar en distintas regiones del país, e incluso, en lugares remotos del mundo. Es importante para una empresa saber qué costos logísticos se tienen en almacenamiento de materia prima, producto semielaborado y producto terminado, ya que según el comportamiento de estos inventarios, se pueden tener posibles combinaciones para evitar que se generen los cuellos de botella, y así, tratar de reducir al máximo los costos que intervienen en el proceso. Aplicando métodos ingenieriles podemos descubrir e implementar técnicas que ayudan a tener un control sobre los procedimientos $\mathrm{y}$, del mismo modo, proyectarse para posibles mejoras que se convierten en ganancias económicas como procedimentales para la compañía.

\section{JUSTO A TIEMPO}

La metodología Justo a Tiempo es una herramienta que, principalmente, se encarga de garantizar que las cosas estén en el lugar adecuado, en el momento adecuado y con las cantidades apropiadas (1). Esto, con el fin de que el cliente pueda satisfacer la necesidad que tiene sin ningún inconveniente. Básicamente, esta filosofía se fundamenta en la calidad de los productos y servicios, reducción de residuos, menores costos, aumento de la productividad y un nivel alto de satisfacción al cliente. El método Justo a Tiempo se empieza a aplicar en los años 70 por la empresa Toyota, debido a la crisis económica que surgió en esta época.

Entre las prácticas de Justo a Tiempo podemos encontrar:
- Control de calidad óptimo.

- Personal calificado.

- Inventarios reducidos.

\subsection{CONTROL DE CALIDAD}

Es necesario para la empresa realizar un adecuado control de calidad, ya que si se filtran productos al mercado en mal estado, esto puede originar que el nombre de la compañía quede en entredicho por la insatisfacción del cliente con el estado del producto; por lo cual, es indispensable montar un sistema de control de calidad a lo largo del proceso productivo, tratando de ajustar los elementos lo más que se pueda con el fin de optimizar y mejorar cada día más el proceso. Es primordial vender un producto que sea de gran utilidad, y que le guste a las personas, ya que esto lleva al crecimiento de la empresa. Entre más satisfacción encuentre el cliente, el nivel de ingresos será mayor.

\subsection{PERSONAL CALIFICADO}

El personal calificado es quizás una de los pilares que ayudan en la construcción y progreso de una empresa; por consiguiente, estos son los que toman las decisiones sobre el producto en proceso y producto terminado. A lo largo de la cadena de abastecimiento se necesitan de personas que tengan el conocimiento de lo que deben hacer en el proceso, personal capaz de realizar una labor específica y global, personas que sean aptas para identificar problemas y posibles soluciones, porque es de allí donde parte la base para estabilizar la empresa. Quizás más adelante logren un crecimiento tanto personal como empresarial.

\subsection{INVENTARIOS REDUCIDOS}

Los inventarios reducidos son quizá uno de los elementos más importantes en la metodología Justo a Tiempo, de ahí que tener inventarios altos implica mayores costos de almacenamiento, y tenerlos muy bajos implica riesgo de escasez, que obligarán posiblemente la compañía a sacar un agotado del producto, lo que en el mercado sería fatal. Para poder tener los inventarios mínimos es necesario seleccionar un proveedor que pueda cumplir con los requerimientos nece- 
sarios para la fórmula del Justo a Tiempo, entre las características que debe reunir el proveedor están: la disponibilidad de producto, ubicación estratégica (en lo posible cercana a la planta), atención inmediata, equipos necesarios para el transporte efectivo y, adicionalmente, otros elementos que son necesarios identificar.

\section{INVENTARIOS}

Las organizaciones manufactureras, generalmente, tienen inventarios, la administración adecuada o no de estos pueden llevarlas al éxito o al fracaso (2), estos están constituidos por materias primas, productos en proceso, suministros o materiales que se utilizan en las operaciones y los productos terminados. Son utilizados en cuestiones tan sencillas como un accesorio o herramientas empleados como parte de un programa de mantenimiento, o algo más complejo, como una combinación de materias primas o semielaborados, que forman parte de un proceso de producción (3). En relación a lo anterior, podemos ver que algunos son inevitables. Todas o, cuando menos, una gran parte de las empresas de sector manufacturero tienen en los inventarios uno de los activos en circulación más representativos financieramente dentro de la organización, esto debido al valor monetario y costos que representan para las mismas. Por esto, la relevancia de la importación y de contar con el articulo adecuado, en la cantidad, lugar y momento adecuado.

\subsection{IMPORTANCIA DE LOS INVENTARIOS}

Para la gran mayoría de las compañías, el stock, como se mencionó anteriormente, es el mayor de los activos circulantes. Cuando una empresa falla en la administración eficiente de sus recursos y se queda sin estos, se pueden presentar agotados, bajos niveles de servicio e insatisfacción del cliente (4). Si la empresa es una tienda de menudeo, el comerciante pierde la utilidad bruta de este artículo; por el contrario, si una firma mantiene niveles excesivos de estos, el costo de mantenimiento adicional puede representar la diferencia entre utilidades y pérdidas. En conclusión, la administración eficiente de los inventarios puede tener un gran impacto en la cadena de valor de la compañía.

\subsection{DECISIONES SOBRE LOS INVENTARIOS}

Generalmente, las personas encargadas de administrar los inventarios en las empresas, siempre se verán enfrentados a decisiones de abastecimiento de los recursos necesarios para las diferentes funciones o procesos de la compañía (5). Estas decisiones se ven resumidas en dos grandes interrogantes.

- ¿Qué cantidad de un artículo ordenar cuando el inventario se va a reabastecer?

- ¿Cuándo reabastecer el inventario de ese artículo?

- ¿Cuál es el mejor valor para adquirir estos artículos?

\section{CADENA DE ABASTECIMIENTO}

La Cadena de Abastecimiento (Supply Chain), incluye todas las actividades relacionadas con el flujo de materiales, productos, dinero, información necesaria para la transformación de materias primas en bienes y productos; en conclusión, la cadena de abastecimiento o suministro es el conjunto de actores o actividades que se encargan de la ejecución de la logística.

De otro lado, engloba los procesos de negocio, las personas, la organización, la tecnología y la infraestructura física que permite la transformación de materias primas en productos, y servicios intermedios que son ofrecidos y distribuidos al consumidor para satisfacer la demanda (6). La gestión eficiente de ésta permite una mejor efectividad en el servicio al cliente y de la cadena de valor, a través del trámite de flujos de información, de artículos y monetario. Por estas razones puede buscarse el éxito ante los competidores en los mercados vigentes, gracias al resultado que produce la conjunción de los objetivos de la cadena de suministro y la implantación de mejores prácticas en áreas como la planificación del suministro y la demanda, producción, transporte, almacenaje, compras y servicio al cliente (7)

\subsection{OBJETIVO DE LA CADENA DE ABASTECIMIENTO}

El principal objetivo del departamento logístico y la cadena de abastecimiento de una compañía 
es abastecer los materiales necesarios en cantidad, calidad y tiempos requeridos al menor costo posible, con el fin de dar un mejor servicio al cliente (8). Es por esto que se hace indispensable una eficiente administración de la cadena de suministro, y se busca la integración óptima de las actividades y actores que intervienen en los procesos logísticos de la empresa para alcanzar una ventaja competitiva sostenible.

\subsection{CARACTERÍSTICAS DE LA CADE- NA DE ABASTECIMIENTO}

- Es dinámica e implica un flujo constante de información, productos y fondos entre las diferentes etapas.

- El cliente es parte primordial de las cadenas de suministro. El propósito fundamental de las cadenas de suministro es satisfacer las necesidades del cliente.

- Una cadena de suministro involucra flujos de información, fondos y productos.

- Una cadena de suministro típica puede abarcar varias etapas que incluyen: clientes, detallistas, mayoristas distribuidores, fabricantes, proveedores de componentes y materias primas.

- Cada etapa de la cadena de suministro se conecta a través del flujo de productos, información y fondos

- El diseño apropiado de la cadena de suministro depende de las necesidades del cliente, como de las funciones que desempeñan las etapas que abarca.

\section{PLANEACIÓN DE COMPRAS Y SUMI- NISTROS (REABASTECIMIENTO).}

Las empresas establecen técnicas para predecir sus necesidades de productos basadas en la demanda. La predicción de los niveles de inventario y del reabastecimiento de existencias que son vitales en procesos donde la demanda depende de diversos factores.

El proceso de compra o reabastecimiento implica la adquisición de materias primas, suministros y componentes para la organización, involucrando decisiones que afectan los costos logísticos, como son las relacionadas con la selección de los puntos de envió del proveedor, la determinación de las cantidades de compra, el momento oportuno del flujo de suministro, la selección de la forma y los métodos de transportación son algunas de las más importantes (9).

\section{DESCRIPCIÓN DEL PROBLEMA}

En la actualidad se presenta un problema en la empresa de bebidas, ya que con el fin de no tener problemas de producción y faltantes en productos terminados para sus clientes, se manejan unos inventarios demasiado altos, en cada una de las 20 plantas que conforman la compañía como tal; por esto, es necesario tratar de ajustarlo un poco, porque se está incurriendo en altos costos de almacenamiento y se está perdiendo espacio que se podría utilizar en otras labores de la compañía; incluso, hasta alquilarlo o venderlo para que sea utilizado por otra empresa. Por tanto se pretende plantear un modelo basado en la teoría con el fin de aplicarlo y lograr resultados exitosos en la empresa. Antes de la solución del problema vamos a hablar un poco de la empresa.

\subsection{GENERALIDADES}

La compañía de bebidas, en la que vamos a trabajar con nuestro método posee una infraestructura física que permite atender las exigencias del mercado, que la convierte en una de las empresas líderes del país, actualmente para cumplir con las necesidades del mercado, la empresa cuenta con 20 plantas productoras ubicadas estratégicamente en el territorio nacional, las cuales suplen las necesidades a lo largo y ancho del país, con ayuda de más de 70 CEDI's que conforman el sistema de distribución.

Para cumplir con los requerimientos del mercado, la compañía cuenta con un grupo de Divisiones que sirven como columna vertebral a todas las actividades que conforman la cadena de valor de la compañía. En la División Nacional de Logística y Producción se llevan a cabo tareas de planeación, organización, dirección, coordinación y control para una eficiente administración de la cadena de suministro; se enfoca en tres grandes campos de trabajo: coordinación de inventarios, coordinación de producciones, coordinación de empaque y producto terminado, y exportaciones. 


\subsection{PLANEACIÓN Y ABASTECIMIEN- TO DE AZÚCAR}

Esta programación se realiza mediante una revisión periódica de los inventarios, la cual se efectúa cada semana el día viernes, en esta se tiene en cuenta: los despachos que se tengan pendientes, inventarios con los que inicia el día cada planta en el almacén, viajes en tránsito y los inventarios de seguridad que se deben mantener, los cuales pueden variar dependiendo del tiempo de entrega, el cual es diferente para cada una de las regiones del país. Es importante tener presente que los ingenios proveedores del azúcar se encuentran ubicados en el Valle del Cauca.

Tabla No 1. Control de Azúcar.

\begin{tabular}{|c|c|c|c|c|c|}
\hline $\begin{array}{c}\text { INVENTARIO } \\
\text { PLANTA KgS }\end{array}$ & $\begin{array}{c}\text { TRANSITO } \\
\text { KgS }\end{array}$ & $\begin{array}{c}\text { SALIDA } \\
\text { Kgs/DÍA }\end{array}$ & $\begin{array}{c}\text { DURACIÓN DE } \\
\text { INV EN PLANTA } \\
\text { (DÍAS) }\end{array}$ & $\begin{array}{c}\text { DURACIÓN DE } \\
\text { INV PLANTA + } \\
\text { TRANS(DÍAS) }\end{array}$ & $\begin{array}{c}\text { DURACIÓN } \\
\text { INV } \\
\text { TOTAL(DIAS) }\end{array}$ \\
\hline 137,900 & & 19,000 & 7.3 & 7.3 & 7.3 \\
\hline 273,000 & 210,000 & 45,000 & 6.1 & 10.7 & 10.7 \\
\hline 103,750 & & 12,000 & 8.6 & 8.6 & $\mathbf{8 . 6}$ \\
\hline
\end{tabular}

Esta tarea es muy importante, y en la que se requiere mucha dedicación, porque se manejan los movimientos y el inventario de la materia prima más importante de la compañía: "el azúcar", sin la cual ninguna planta puede producir, pues es el principal ingrediente del Jarabe Simple, además de representar el mayor costo para el almacén general, por su valor monetario.

Para el abastecimiento de azúcar es necesario tener en cuenta las épocas del año, ya que, por ejemplo, en épocas como diciembre el país consume demasiado líquido. Por consiguiente, los despachos desde el ingenio hacia las plantas son mas seguidos, ya que, como sube el consumo promedio de las plantas, deben realizarse despachos con menos espacio de tiempo. En temporada de invierno, por cuestiones de derrumbes en la carretera y mal estado de las vías, es necesario hacer los envíos por lo menos con un día de anticipación a lo requerido, para no sufrir ningún contratiempo con la producción En vacaciones escolares también se aumenta un poco el consumo.

\subsection{RECOLECCIÓN DE LA INFORMACIÓN YDATOS}

Mediante el sistema ERP (SAP), se manejan transacciones de inventarios que permiten visualizar los stock de materia prima, así como los consumos realizados (esta información está disponible para la compañía desde el 2001), la cual permite realizar análisis y establecer la salida diaria (consumo) o mensual para cada planta, como también a nivel del país; de igual forma, se puede conocer la cantidad con que se inicia el día.

Para garantizar una mayor confiabilidad de los datos, vía correo electrónico, se maneja el intercambio de información con cada jefe de almacén de cada planta del país, solicitando la cantidad de existencia actual de azúcar, así como la salida (consumo) diario, ya que esto puede variar según planes de producción para cada semana (esta demanda puede variar debido a las fluctuaciones de las ofertas en el mercado, apoyos realizados a otras plantas, saneamiento, paradas de líneas de producción, mantenimientos programados, entre otros factores).

Igualmente, se recibe por parte de los ingenios una notificación diaria de los despachos realizados, esto con el objetivo de realizar un seguimiento a la programación efectuada semanalmente a fin de garantizar una gestión efectiva de esta materia prima.

\subsection{TABULACIÓN,ANÁLISIS Y DESARROLLO}

Mediante el programa Excel, se elabora macros (tablas), las cuales permiten manejar la información de SAP y de los almacenes de materia prima, de modo que se puedan llevar controles diarios y se facilita, eficientemente, el proceso de programación del abastecimiento de los inventarios semanalmente. Estas macros se construyeron bajo parámetros ya establecidos por la compañía.

Políticas de stock de seguridad: estas políticas establecen el stock de seguridad para cada planta, de acuerdo a su ubicación en el territorio nacional, así como su capacidad de producción según sus líneas (maquinas), e igualmente, su capacidad almacenamiento. Esto con el fin de tener una pronta respuesta en caso de eventualidades o urgencias. 
Las macro, desarrolladas para el control, seguimiento, análisis y programación del abastecimiento, manejan las siguientes variables básicas, para un desarrollo efectivo de la tarea logística:

Planta y Ubicación: Nombre de la Planta embotelladora y la zona del país donde se encuentra ubicada.

Tipo de Azúcar: Hay plantas que manejan varios tipos de azúcar, según su producción, entiéndase por TF (azúcar Sulfitada), LF (azúcar licor fino); por otra parte, todas manejan azúcar refinada y es la materia prima de mayor consumo.

Inventario en Planta: Es la cantidad de inventario actual - kilogramos de azúcar que existe en la planta de estudio.

Pendientes por despachar: son los vehículos en $\mathrm{kg}$ de azúcar pendientes por despachar de la programación enviada, los vehículos son estándar por peso y solo pueden transportar 700 quintales equivalentes a $35.000 \mathrm{~kg}$ (un quintal es la equivalencia de un bulto de $50 \mathrm{~kg}$ ).

Inventario en tránsito: son los vehículos ya despachados que se encuentran cumpliendo con el recorrido para entregar la materia prima; para el inventario en tránsito se toma en cuenta los KG trasportados por dicho vehículo. El tiempo de transito intervienen en la decisiones de las políticas de inventario.

Salida día promedio: es la cantidad de azúcar en $\mathrm{kg}$ que se consume diariamente en la planta.

Días de Stock en planta: son los días que dura la existencia de azúcar, según el consumo diario de la misma. Se calcula como:

$$
\text { Días de Stock }=\frac{\text { Inventario en Planta }}{\text { Salida Promedio }}
$$

Días de Stock total: son los días de inventario de azúcar, que incluye disponibilidad en planta $\mathrm{y}$ el inventario en tránsito divido entre el consumo diario.

Necesidad: es la cantidad de azúcar semanal que necesita la planta. Se programa el cálcu- lo en la macro, de esta necesidad, teniendo en cuenta las variables de políticas de inventario, el inventario en planta, los pendientes por despachar y el inventario en tránsito:

Días de Stock $=\frac{\text { Inv.Planta }+ \text { Inv.Transito }}{\text { Salida Promedio }}$

Días de inventario de seguridad: que se establece según parámetros preestablecidos por la compañía, y varía según el tiempo de tránsito entre el ingenio y cada una de las plantas.

Despacho programado: es la aproximación de las necesidades al múltiplo superior más cercano de 700 quintales. Esto debido a que por costos de flete se realizan despachos de vehículos con capacidad de carga de 35 toneladas, equivalentes a 700 quintales $(35.000 \mathrm{~kg})$.

\section{DESARROLLO DE LA GESTIÓN DE INVENTARIOS EN UNA EMPRESA DE BEBIDAS}

Para tener en cuenta una adecuada gestión de inventarios en la compañía, se desarrolló un paso a paso que debe ser desarrollado por cuenta de la empresa para no tener ningún percance en la implantación y ejecución del método. Dichas etapas son los siguientes:

\section{Ilustración 1. Fases del Proceso}




\subsection{PLANTEAMIENTO DEL MÉTODO JUSTO A TIEMPO}

Para este caso en especial, se tratará de adaptar un modelo que funcione como el sistema Justo a Tiempo, pero con una serie de condiciones que ayudarán a la compañía de bebidas a no tener problemas de faltantes o retrasos en la producción por falta de materia prima.

Primero que todo, se debe considerar que para plantear un sistema Justo a Tiempo, es necesario que tanto el proveedor como la empresa encargada del trasporte tengan respuesta inmediata ante cualquier eventualidad, aunque se tiene planeado programar semanalmente los despachos de azúcar, esta información llegaría a los proveedores, por medio de unos cuadros tipo diagrama de Gantt, con un día de anticipación, al comienzo de la siguiente semana, con el fin de que estén preparados para los pedidos de la siguiente semana.

\subsubsection{Condiciones a tener en cuenta para el abastecimiento:}

- Las plantas en una semana sin alteraciones, normalmente, producen las bebidas en dos turnos, cada uno de ocho horas.

- Cuando las plantas tienen paradas por mantenimiento, éstas, generalmente, producen mínimo la producción que necesitan para cuatro días de parada.

- Tener en cuenta que las plantas sí aumentan un poco la velocidad de las maquinas, sin afectar la eficiencia de éstas; e igualmente, intensificado los turnos que sean de 24 horas, se pueden gastar en dos días lo que se consumen en cuatro.

- El tránsito de un vehículo con azúcar, según el territorio donde se encuentra la planta, puede realizarse en horas; como para otros puede ser días.

- Para calcular los días de inventario que debe tener mínimo una planta, se debe tomar la opción de una parada sin previo aviso (mantenimiento inmediato) y, además, los días en tránsito que se demora en llegar un vehículo desde el ingenio.
Como se menciona anteriormente, esto bajaría los costos de inventario, ya que se pasaría de tener entre 16 y 20 días de inventario en la planta más lejana, con respecto al ingenio, a 7 y 9 días aproximadamente.

\subsubsection{Capacitación para las personas involucradas:}

Es vital que el personal de la compañía conozca este proyecto, ya que desde que se realizan las pruebas, los empleados juegan un papel fundamental en el plan, por consiguiente una de las fases iniciales es darle instrucciones a las personas involucradas, que para este caso serían: el gerente de la planta, jefe de almacén, auxiliares de almacén, división nacional de logística y división nacional comercial, áreas involucradas en el correcto funcionamiento del Justo a Tiempo. Básicamente la información que se transmite de las plantas al programador es la más importante, ya que es necesario que una vez llegue el vehículo con azúcar, sean ingresadas al sistema, la información de las cantidades recibidas; así también otras informaciones adecuadas como lo es el número de la factura, nombre del conductor, empresa trasportadora, placas del vehículo, esto con el fin de validar todos los datos enviados con el ingenio y borrarlo del inventario en tránsito. Podemos mencionar que el proceso que debe realizar el programador de la división de logística es el siguiente: semanalmente se realiza la programación del azúcar mediante los datos generados por la ERP (SAP), la información recopilada de las plantas (inventario actual y consumo diario); y finalmente, esta información se introduce en el archivo de Excel, luego por medio de un diagrama de Gantt se procede a marcar el número de viajes de azúcar que serán enviados para la planta, en el cajón correspondiente al día que se debe enviar, este día se escoge teniendo en cuenta los días de inventario actual y los viajes en tránsito.

Tabla 2.

\begin{tabular}{l} 
DISTRIBUCION SEMANAL DE AZUCAR \\
SEMANA DEL 22 AL 29 DE NOVIEMBRE DE 2013 \\
\cline { 2 - 8 } \\
\cline { 2 - 8 }
\end{tabular}




\subsubsection{Pruebas en las plantas:}

Después de haber planeado el proyecto y capacitado el personal sobre las labores que se deben realizar para una eficiente gestión de inventarios; es necesario, antes de implantar el proyecto, escoger una planta donde se realizarán los ensayos, porque, de ahí en adelante, esta será un punto de partida para analizar posibles fallas e ir corrigiéndolas antes de montar el sistema en todas las plantas. Para estas pruebas se aconsejaría seleccionar un planta que esté en un rango medio; es decir, que no sea una de las grandes productoras, e igualmente, que no sea una de las pequeñas. En el caso de las pequeñas, por su poco manejo de inventarios; y en el caso de las grandes, por su alta fluidez y entrada de material. El término medio se define con el fin de verificar todos los escenarios posibles, por lo que ésta, a veces, según la temporada, tiene consumo alto, o en otra tiene consumo bajo; lo que daría posibilidad de realizar ajustes al modelo si se identifica la temporada; además, se podrían analizar posibles cambios con el fin de adaptar un sistema de clasificación (Megaplanta, planta y microplanta) que pueda mostrar cómo se pueden manejar las plantas según su categoría.

\subsubsection{Mejoramiento del planteamiento:}

Con base en las pruebas se identifican errores que pueden ser corregidos, a medida que se avanza en el proceso, y se trata de establecer métodos puntuales que puedan adaptarse a sistemas de gestión más eficientes, modificaciones en el proyecto y objetivos puntuales que puedan también, tener alguna influencia en el método planteado. En este proceso se deben analizar las metas claras planteadas inicialmente e identificar otras que se puedan ir presentando en el transcurso del proceso. Por otra parte, como se proponen estudios periódicos que puedan evaluar el funcionando del sistema, con el fin tener un mejoramiento continuo del proyecto; igualmente, analizar si es posible implantar dicho método en todas las plantas o si solo funcionara en algunas.

\subsubsection{Implantación del Justo a Tiempo:}

En esta etapa del proceso se puede analizar todo el trabajo que se ha venido haciendo, como son las pruebas, los mejoramientos y la capacitación, de modo que se note una integración de manera ideal con cada uno de estas etapas; en la implantación del método Justo a Tiempo, podemos encontrar variaciones en los costos que muy probablemente disminuirán a medida que se vaya ajustando el proyecto:

Costos de consecución: estos costos se verán reducidos debido a que no se pedirá tanta azúcar como se pedía comúnmente, en consecuencia a esto se pasara de tener entre 12 y 15 días de inventario a tener en las plantas entre 7 y 9 días de inventario; estas operaciones reducirían los costos ingresados mensualmente por materia prima en cada planta, los cuales se verían reflejados en los cierres contables que se hacen mensualmente, por consiguiente cada uno de los centros productores podría aumentar los indicadores de eficiencia, y subir sus indicadores de gestión, por lo cual podrían subir de posición en el escalafón mensual que se realiza en oficina central con el fin de ir notando el mejoramiento de las plantas.

Costos de agotamiento: El costo por faltante puede generarse por no tener suficiente material para cumplir con lo pedido, es decir por parte de producción si no hay materias primas para elaborar la bebida se entraría a facturar un costo por faltante, pero este es quizás menos complejo porque puede haber inventario del producto en empaque y producto, si es más relevante cuando el pedido es realizado para un producto terminado, ya que al no llegar este producto al tendero, puede considerarse como un posible lio, esto debido a que el tendero le comunicara al consumidor que no tiene la bebida porque está agotada , lo cual desencadenaría una serie de reacciones por parte del comprador como desconfianza en la empresa, insatisfacción con el producto y hasta una posible migración hacia otro artículo con el cual no tenga la misma satisfacción, pero que si lo pueda tener en el día a día si inconveniente. Este costo debe utilizarse lo menos posible en las compañías, con el fin de no perder clientes.

Costo de almacenamiento: Básicamente el costo de almacenamiento es el costo que se tiene por mantener los materiales almacenados en un lugar, por lo cual se tienen en cuenta los 
valores de alquiler del local, espacio ocupado por el almacén y el espacio que ocupa cada materia prima en el almacén, teniendo discriminada esta información podemos calcular lo que se está gastando actualmente y mirar una vez implantado el sistema en cuanto dinero y espacio se redujo el almacenamiento de la planta.

Costo de Inventario: El costo de inventario se puede sacar agrupando los 3 costos anteriores. Este costo finalmente se puede hallar sumando el costo de consecución (multiplicar el costo unitario de un objeto específico y multiplicarlo por el número de objetos existentes de este en el almacén), costo por faltante o agotamiento y costo de almacenamiento (Espacio ocupado por un artículo multiplicado por el total de artículos existentes en almacén de este).

Después de tener claro todo lo hablado anteriormente podemos entrar a definir los posibles cambios organizacionales que se pueden tener con la implantación del método y compararlo con el sistema que se maneja actualmente.

Cuadro No 1. Comparación de Métodos.

\begin{tabular}{|c|c|}
\hline Actual & JIT \\
\hline $\begin{array}{c}\text { Demasiado espacio } \\
\text { para almacenar materia prima } \\
\text { en inventario }\end{array}$ & $\begin{array}{c}\text { Espacio óptimo para una } \\
\text { adecuada producción de la } \\
\text { planta }\end{array}$ \\
\hline $\begin{array}{c}\text { Altos tiempos en descargue } \\
\text { de azúcar semanalmente }\end{array}$ & $\begin{array}{c}\text { Reducción notable de } \\
\text { descargues en la semana }\end{array}$ \\
\hline Espacio del almacén \\
totalmente copado & $\begin{array}{c}\text { Un poco de más espacio para } \\
\text { transitar por el almacén o } \\
\text { hasta alquilar espacios } \\
\text { innecesarios }\end{array}$ \\
\hline
\end{tabular}

Las características descriptas en el cuadro son las que inicialmente se tiene presupuestada atacar, pero posiblemente se pueden dar más ventajas con el sistema Justo a Tiempo que con el método que se encuentra vigente.

\section{Ejecución del Justo a tiempo:}

En la ejecución del justo a tiempo se debe velar porque se ejecute cada uno de los procesos de manera conjunta y efectiva, ya que cualquier equivocación o falta de un paso en el desarrollo puede dejar a la empresa mal parado el proyecto.

En la confección del proceso primero que todo tiene que haber compromiso totalmente de los entes involucrados, como lo son el personal de almacén de cada una de las plantas, el personal logístico que se encarga de la programación desde oficina central, los proveedores y los transportadores; con respecto al personal de la compañía se debe garantizar que la ERP este actualizada con los viajes de azúcar que le llegan y las cantidades, por parte del programador tiene que estar atento a los tránsitos, el stock de seguridad óptimo para cada planta y por supuesto la programación de los viajes según el día indicado para el abastecimiento, por el lado de los proveedores es necesario tener la materia prima disponible en cualquier momento, ya que si es necesario algún viaje de urgencia este se deberá realizar sin ningún problema y por último la empresa transportadora debe cumplir con los tiempos de transporte establecidos.

\section{RECOMENDACIONES}

El método justo a tiempo a pesar de que ahorrar costos y tiempos en la compañía de bebidas, también tiene que ser bien administrado, ya que en épocas como el invierno es muy dificultoso el tránsito, ya que hay derrumbes e inundaciones, por lo cual el tiempo en tránsito de un vehículo es casi el 1.5 o hasta 2 veces más que lo normal y en temporadas de alto consumo por ejemplo diciembre los consumos son muy altos por que se aumenta la venta de bebidas en el país.

\section{CONCLUSIONES}

- Ofrecer un abastecimiento continuo y oportuno según el desarrollo herramientas de estudios y análisis adecuados, permitirá afrontar sin agotados o escases de materia prima temporadas críticas en la operaciones logísticas para la compañía, como es la temporada navideña y vacacional. Esto debido a las múltiples restricciones en las actividades logísticas del país.

- La administración y control eficiente de las actividades logísticas de abastecimiento de los inventarios de la cadena de suministro de la empresa, permitirá racionalizar eficientemente el uso del espacio en los almacenes, manejara niveles de inventarios adecuados a los procesos 
PROPUESTA METODOLÓGICA PARA LA GESTIÓN DE INVENTARIOS EN UNA EMPRESA DE BEBIDAS POR EL MÉTODO JUSTO A TIEMPO. CASO DE ESTUDIO: ABASTECIMIENTO DE AZÚCAR.

productivos, consolidara eficientemente el carga y descargue de materiales, todo esto generara una disminución en los costos financieros de la compañía producto de las actividades logísticas, así como una mejora en la planeación de la producción de materiales de nuestro proveedor, disminuyendo el riesgo de almacenamiento de inventarios innecesarios.

- Es fundamental la adecuada interacción entusiasta, responsable y profesional me- diante una comunicación efectiva con las diferentes divisiones inherentes en el proceso productivo y de expansión de la organización, se debe contar con el apoyo de comercial, mercadeo y ventas, que permitan alcanzar los objetivos de la compañía.

- Por medio de la propuesta metodológica, se pretende que algunas empresas puedan tener una ayuda para plantear un efectivo manejo de inventarios que les reducirá los costos y aumentar sus ingresos.

\section{BIBLIOGRAFÍA}

1. Just in Time: El sistema de producción Justo a Tiempo. Arndt, Philipp . Murcia: s.n., 2005. Seminario del Curso XXX. pág. 2 .

2. Longenecker, Justin, Moore, Carlos y Palich, Leslie. Administración de pequeñas empresas. Mexico D.F.: CENGAGE Learning, 2010.

3. Muller, Max. Fundamentos de administración de inventarios. Bogota: Norma, 2004.

4. Muñoz Negron, David. Administración de operaciones. Enfoque de administración de procesos de negocios. Mexico D.F.: CENGAGE Learning, 2009.

5. EAFIT (David Fernando Bernal Rodriguez). Consultorio Contable. [En línea] [Citado el: 29 de Noviembre de 2013.] http://www.eafit.edu.co/escuelas/administracion/consultorio-contable/Documents/Nota $\% 20 \mathrm{de} \% 20$ clase $\% 2019 \% 20$ Justo\%20a\%20tiempo.pdf.

6. Escuela de Administración de Negocios. University of Texas. 2009, E.A.N, págs. 45-49.

7. Salazar Davila, Fernando. DIAGNOSTICO Y SCOR MODEL PARA LA GESTIÓN DE LA CADENA DE SUMINISTRO DE LA EMPRESA MANGUERAS INDUSTRIALES. Santiago de Cali: UNIVERSIDAD AUTÓNOMA DE OCCIDENTE, 2009.

8. Chavez, Jorge y Torres, Rodolfo. Supply Chain Management (Gestión de la cadena de suministro). Providencia (Chile): RIL Editores, 2012.

9. Chapman, Stephen. Planificación y control de la producción. Mexico D.F.: Pearson Educación, 2006.

10. Ballou, Ronald. Logística: administración de la cadena de suministro. Mexico D.F.: Pearson educación, 2004. 